\title{
Two-radii 8APSK and Two-radii 16APSK Modulations as Alternatives to 8PSK and 16QAM
}

\author{
Dayan Adionel Guimarães
}

\begin{abstract}
In regard to the $M$-ary phase-shift keying (MPSK) modulation and the quadrature amplitude modulation (MQAM), $M P S K$ is preferred up to $M=8$, with $M Q A M$ being the most common choice for $M>8$. In this letter, the modulations tworadii 8-ary and 16-ary amplitude-phase shift keying (APSK), respectively named 2 r8APSK and 2 r16APSK, are proposed as alternatives to 8PSK and square 16QAM, in this order. The 2r8APSK achieves higher power efficiency than the 8PSK, at the cost of a higher peak-to-average power ratio (PAPR) and a slightly more complex receiver. The 2r16APSK yields lower PAPR than the 16QAM, with roughly the same receiver complexity, but at the cost of a slightly lower power efficiency. Comparisons with other APSK-based modulations are made as well.
\end{abstract}

Keywords-Amplitude-phase shift keying, digital transmission, phase-shift keying, quadrature amplitude modulation

\section{INTRODUCTION}

$\mathbf{T}$ HE $M$-ary phase-shift keying ( $M$ PSK) modulation and the $M$-ary quadrature amplitude modulation (MQAM) are widely used in digital communication systems. Both have the same spectral efficiency, but the MPSK has the advantage of lower receiver complexity, since there is no need for estimating any threshold or received signal levels, thanks to the equal-energy symbols. However, MQAM receivers have an inherent higher complexity because the average received symbol energy must be estimated to establish proper threshold levels or equivalent quantities, as the consequence of having to deal with different symbol energies [1, p. 441]. On the other hand, the MQAM achieves higher average Euclidean distance between its constellation symbols than the MPSK, for the same average symbol energy, yielding higher power efficiency, i.e., lower bit error rate (BER) at a fixed signal-tonoise ratio. Hence, for $M>8$ it is worth paying for a higher receiver complexity of the $M \mathrm{QAM}$ with respect to the $M \mathrm{PSK}$, since large performance gains are attained by the former. The power efficiency of the 8QAM is approximately $2 \mathrm{~dB}$ higher than in the case of the 8PSK, but the lower receiver complexity of the latter justifies its adoption in the majority of situations. In summary, the MPSK is the preferred solution up to $M=8$; the $M$ QAM is often the choice for $M>8$.

The modulation selection scenario becomes clumsier if another important metric is also taken into account: the peakto-average power ratio (PAPR). As the name suggests, it is the ratio between the peak signal power and its average power.

Dayan A. Guimarães is with the Instituto Nacional de Telecomunicações (Inatel), Santa Rita do Sapucaí - MG - Brazil. Tel:+55 (35) 3471 9227, e-mail: dayan@inatel.br. This work was partially supported by Finep, with Funttel resources, Grant 01.14.0231.00, under the Inatel's project Centro de Referência em Radiocomunicações (CRR). DOI: 10.14209/jcis.2018.30.
Signals having non-constant envelope are special candidates to exhibit high PAPR, making it difficult the project of large dynamic range and power-efficient amplifiers. This is owed to these main reasons: i) a large amplifier back-off must be set if it is required that the signal does not reach the amplifier nonlinear region in order to be undistorted; however, a low power efficiency is expected as a consequence of the large backoff; ii) if the amplifier operates in its nonlinear region, higher power efficiencies can be attained, but the amplified signal is distorted and suffers from spectral regrowth [1, p. 441], which ends up widening the transmitted signal bandwidth. Moreover, even constant-envelope signals, like MPSK, exhibit non-constant envelope after filtering. On the other side, the envelope fluctuations of unfiltered MQAM signals are inherent to the technique, but are further increased due to filtering.

This letter adds two new modulations to the above scenario, allowing for an increased degree of freedom when choosing between an 8-ary or a 16-ary two-dimensional modulation scheme. The new modulations are special cases of the broad family of amplitude-phase shift keying (APSK) modulations [2], to which the MQAM also belongs. The constellation of each proposed modulations is formed by two concentric rings with different radii, with $M / 2$ symbols placed equally spaced on one ring and the remaining $M / 2$ symbols placed equally spaced on the other ring. Hence, each constellation looks like two concentric $\frac{M}{2}$ PSK constellations. The proposed modulations are named two-radii 8-ary amplitude-phase shift keying (2r8APSK) and two-radii 16-ary amplitude-phase shift keying (2r16APSK). The targeted application of the 2r8APSK and 2r16APSK modulations is to serve as alternative choices with respect to the 8PSK and the square 16QAM, respectively.

It must be emphasized that constellation designs based on the generic APSK principle are not new [2]. Directly or indirectly motivated by this design strategy, many constellation alphabets have been proposed, developed under a variety of optimization criteria [3]-[7]. Table I summarizes exemplifying proposals, allowing for establishing the differences between them and the constellations developed herein.

The remainder of the letter is organized as follows: Section II describes the proposed modulations and the transmitter and receiver structures. Sections III, IV and V are devoted to the numerical results. The conclusions are drawn in Section VI.

\section{Proposed Modulations}

Let the unit-energy signal-vectors (constellation symbols) of an MPSK modulation be written as

$$
\mathbf{s}_{i}=\left[\begin{array}{c}
\cos [2(i-1) \pi / M] \\
\sin [2(i-1) \pi / M]
\end{array}\right]
$$


TABLE I

MAIN CHARACTERISTICS OF THE 2RMAPSK AND OTHER APSK-BASED SCHEMES. CFM, SEP, BEP AND RRC STAND RESPECTIVELY FOR: CONSTELLATION FIGURE OF MERIT, SYMBOL ERROR PROBABILITY, BIT ERROR PROBABILITY, ROOT RAISED COSINE.

\begin{tabular}{|c|c|c|c|c|c|c|}
\hline Reference & $\begin{array}{c}\text { Modulation } \\
\text { order }\end{array}$ & $\begin{array}{l}\text { Optimization (O) or } \\
\text { design (D) criterion }\end{array}$ & $\begin{array}{c}\text { Constellation } \\
\text { shape }\end{array}$ & $\begin{array}{c}\text { PAPR } \\
\text { analysis? }\end{array}$ & $\begin{array}{c}\text { RRC } \\
\text { filtered? }\end{array}$ & $\begin{array}{c}\text { Performance } \\
\text { metric }\end{array}$ \\
\hline This letter & 8,16 & Minimum BEP $(\mathrm{O})$ & Two-radii $M$ APSK & Yes & Yes & $\begin{array}{c}\text { CFM and a tight } \\
\text { upper bound on BEP }\end{array}$ \\
\hline$[2]$ & Several & Empirical (D) & Several shapes & No & No & $\begin{array}{l}\text { Union bound } \\
\text { on SEP }\end{array}$ \\
\hline [3], [4] & 16 , possibly 8 & $\begin{array}{c}\text { Set operations based on } \\
\text { quaternary constellations (D) }\end{array}$ & Asterisk-shaped 16QAM (A16QAM) & Yes & No & $\begin{array}{l}\text { CFM and union } \\
\text { bound on SEP }\end{array}$ \\
\hline$[5]$ & 64 & $\begin{array}{l}\text { Maximum mutual } \\
\text { information }(\mathrm{O})\end{array}$ & $\begin{array}{c}\text { Four-ring APSK with } 4+12+20+28 \\
\text { and } 4+12+16+32 \text { symbols }\end{array}$ & No & No & $\begin{array}{l}\text { Spectral efficiency in } \\
\text { bits per channel use }\end{array}$ \\
\hline [6], [7] & 16,32 & Not addressed & $\begin{array}{l}\text { Two-ring 16APSK with } 4+12 \text { symbols and } \\
\text { three-ring } 32 \text { APSK with } 4+12+16 \text { symbols }\end{array}$ & No & No & $\begin{array}{l}\text { Union bound on } \\
\text { SEP and BEP in [7] }\end{array}$ \\
\hline
\end{tabular}

for $i=1,2, \ldots, M$. These signal-vectors are obviously on a ring with unit radius. Shifting the even-numbered signalvectors to an outer ring with radius $\sqrt{z}$, one obtains

$$
\mathbf{s}_{2 k} \leftarrow \sqrt{z} \mathbf{s}_{2 k},
$$

for $k=1,2, \ldots, M / 2$. To set the average symbol energy to $E$, all signal-vectors must be rescaled according to

$$
\mathbf{s}_{i} \leftarrow \mathbf{s}_{i} \sqrt{E}\left(\frac{1}{M} \sum_{k=1}^{M} \mathbf{s}_{k}^{\mathrm{T}} \mathbf{s}_{k}\right)^{-1 / 2},
$$

where, and hereafter, it is implicitly assumed equally-likely symbols, and where $[\cdot]^{\mathrm{T}}$ denotes vector transposition.

Fig. 1 illustrates the process of generating a 2r16APSK constellation from a 16PSK constellation; the same reasoning applies to the construction of the 2r8APSK constellation from the 8PSK. As $z$ is increased, the resultant PAPR increases, because the difference between the radii of the outer and the inner rings increases. On the other hand, the minimum Euclidean distance between the signal-vectors becomes progressively larger up to a given value of $z$, beyond which this distance starts to diminish. Hence, there is an optimum $z$ for which the bit error probability $P_{\mathrm{b}}$ is minimum. To find this optimum $z$, the union bound $[8$, p. 772$]$

$$
P_{\mathrm{b}} \leq \frac{1}{2 M \log _{2} M} \sum_{i=1}^{M} \sum_{\substack{k=1 \\ k \neq i}}^{M} d_{i, k}^{H} \operatorname{erfc}\left(\frac{d_{i, k}^{E}}{2 \sqrt{N_{0}}}\right)
$$

was applied to the two-radii constellations, for $M=8$ and $M=16$, assuming an additive white Gaussian noise (AWGN) channel. In (4), $N_{0}$ is the power spectral density of the noise, in watts per hertz, $d_{i, k}^{E}=\left\|\mathbf{s}_{i}-\mathbf{s}_{k}\right\|$ is the Euclidean distance between $\mathbf{s}_{i}$ and $\mathbf{s}_{k}$, with $\|\cdot\|$ denoting the Euclidean norm, and $d_{i, k}^{H}$ is the Hamming distance between the binary words into which $\mathbf{s}_{i}$ and $\mathbf{s}_{k}$ are mapped. It is assumed that Gray mapping is applied sequentially to the signal-vectors, starting with the all-zero word for $\mathbf{s}_{1}$. It is noteworthy that, although (4) is an upper bound, it is known to closely approximate the actual $P_{\mathrm{b}}$ for moderate-to-high signal-to-noise ratios.

Since the locations of the signal-vectors depend on $z$, according to (2) and (3), the bound (4) can be written as $P_{\mathrm{b}}(z)$. Fig. 2 shows $P_{\mathrm{b}}(z)$ for the 2r8APSK (left) and the 2r16APSK (right) constellations, from where it can be noticed that the optimum $z$ depends on the average signal-to-noise ratio per bit, $E_{\mathrm{b}} / N_{0}$, where $E_{\mathrm{b}}=E / \log _{2} M$ is the average energy per bit. This dependence is less visible in the case of the 2r16APSK,

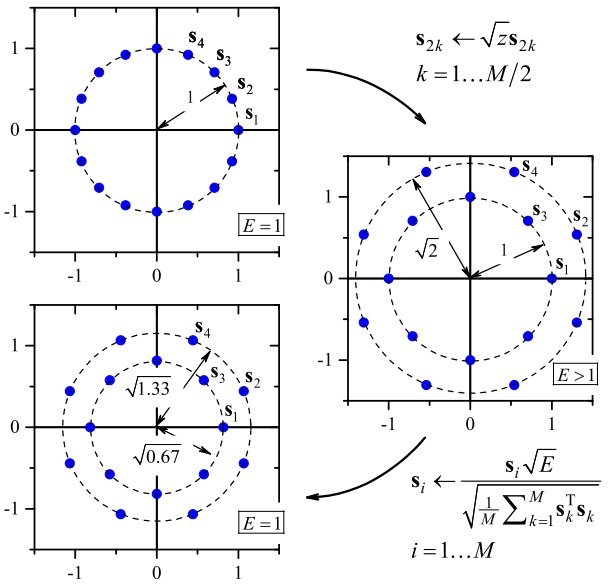

Fig. 1. The process of constructing a 2r16APSK constellation from a 16PSK constellation. The value $z=2$ was adopted just for illustration purpose.

for which the optimum values of $z$ are very close to each other for the three curves shown. The references $E_{\mathrm{b}} / N_{0}=9 \mathrm{~dB}$ and $E_{\mathrm{b}} / N_{0}=12 \mathrm{~dB}$ were respectively chosen, since the resultant bit error probability around $1 \times 10^{-3}$ has practical relevance (recall that the majority of digital communication systems guarantee target error rates not solely by the performance of the modulation scheme, but also by the influence of channel coding, diversity and other performance-improving techniques). For these references of $E_{\mathrm{b}} / N_{0}$, the optimum $z$ is 3.3 for the 2r8APSK and 2.6 for the 2r16APSK.
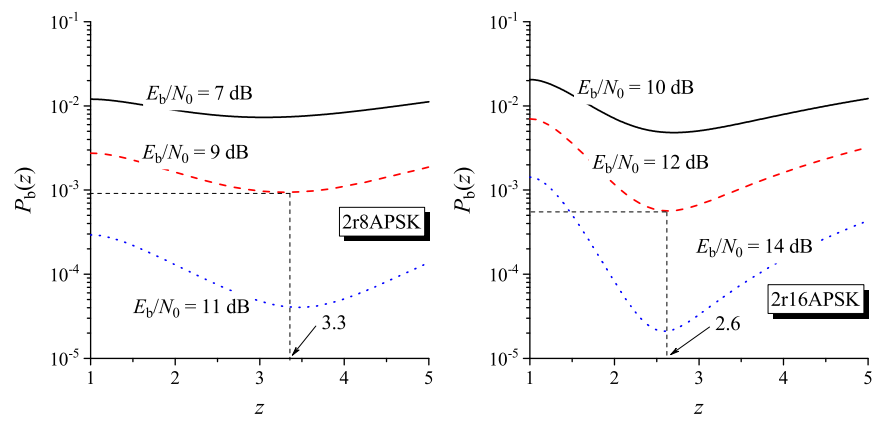

Fig. 2. Bit error probability for the 2r8APSK (left) and 2r16APSK (right) as a function of the ratio $z$ between the outer and the inner symbol energies.

Denoting the energies of the inner and outer symbols respectively as $E_{\mathrm{r} 1}$ and $E_{\mathrm{r} 2}$, the relationship between these energies and the average symbol energy $E$ can be established 
from the relations $E_{\mathrm{r} 2}=z E_{\mathrm{r} 1}$ and $E=\left(E_{\mathrm{r} 1}+E_{\mathrm{r} 2}\right) / 2$, yielding

$$
E_{\mathrm{r} 1}=\frac{2 E}{1+z}, \quad E_{\mathrm{r} 2}=\frac{2 E}{1+1 / z},
$$

which results in $E_{\mathrm{r} 1}=0.4651 E$ and $E_{\mathrm{r} 2}=1.5349 E$ for the 2r8APSK constellation, and $E_{\mathrm{r} 1}=0.5556 E$ and $E_{\mathrm{r} 2}=1.4444 E$ for the 2r16APSK. The final $2 \mathrm{r} 8 \mathrm{APSK}$ and 2r16APSK constellations are depicted in Fig. 3. It is worth highlighting that the distances between nearest-neighbor symbols pertaining to the inner ring are not equal to the distances from these symbols to the nearest ones located on the outer ring, although these distances visually seem to be the same. From simple trigonometry applied to Fig. 3, and using (5), it can be shown that the minimum Euclidean distance is $d_{1,2}^{E}=\{2 E[1-\sqrt{2 z} /(1+z)]\}^{0.5}$ for the 2r8APSK and $d_{1,3}^{E}=[4 E(1-\sqrt{2} / 2) /(1+z)]^{0.5}$ for the 2r16APSK.
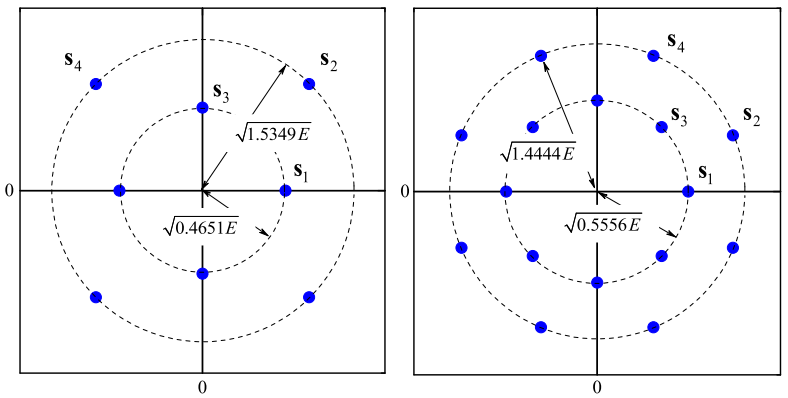

Fig. 3. 2r8APSK (left) and 2r16APSK (right) constellations. The first four signal-vectors are labeled; the other labels go in sequence. Symbol-to-bit mappings follow the 3-bit and 4-bit Gray code, respectively.

The 2rMAPSK modulator block diagram is shown in Fig. 4. In fact, it applies to any two-dimensional modulation scheme whose base functions are $\phi_{1}(t)=\sqrt{2 / T} \cos \left(2 \pi f_{\mathrm{c}} t\right)$ and $\phi_{2}(t)=\sqrt{2 / T} \sin \left(2 \pi f_{\mathrm{c}} t\right)$ [1, p. 440], where $T$ is the symbol duration and $f_{\mathrm{c}}$ is the carrier frequency. If the look-uptable (LUT) is constructed according to Fig. 3, the 2r8APSK or the 2r16APSK modulated signal is generated.

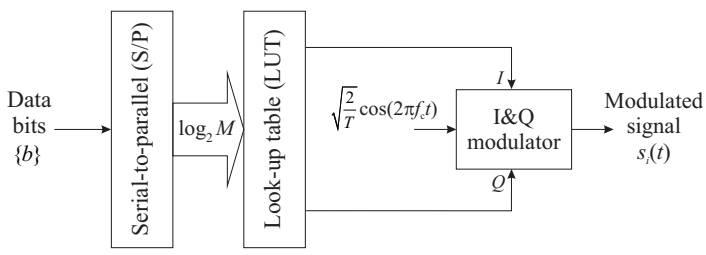

Fig. 4. Modulator block diagram that applies to all modulations in analysis. Only the LUT differs from one modulation to another.

The maximum-likelihood (ML) receiver for the 2r8APSK and the 2r16APSK modulations can be constructed in light of the generalized ML receiver given in [1, p. 418], resulting in the block diagram shown in Fig. 5. The received signal $x(t)$ is correlated with the base functions to form the elements of the observed signal-vector $\mathbf{x}=\left[x_{1}, x_{2}\right]^{\mathrm{T}}$. The inner products between $\mathbf{x}$ and all signal-vectors $\mathbf{s}_{i}$ are performed, and each result subtracts half of the respective received symbol energy, which is determined from (5), according to which constellation ring the symbol belongs. The top $M / 2$ gray-shaded blocks refer to odd-numbered symbols and the bottom $M / 2$ blocks refer to even-numbered symbols. The largest output of these blocks will determine the index of the estimated symbol $\hat{\mathbf{s}}$, which is then mapped into the estimated data bits $\hat{b}$.

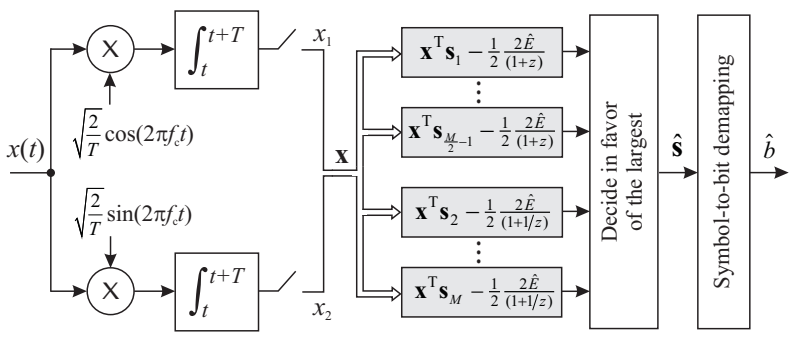

Fig. 5. Receiver block diagram for the 2rMAPSK modulated signals.

Notice in Fig. 5 that the average symbol energy used inside the gray-shaded blocks is the estimate $\hat{E}$ instead of the actual $E$. Modern software-defined radio (SDR) technology allows for simple and accurate computation of $\hat{E}$ through digital signal processing. For instance, the receiver can make use of the feedback loop signal of the automatic gain control (AGC) mechanism to estimate $E$. This AGC is of paramount importance to keep the received signal excursion within the dynamic range of the analog-to-digital converter (ADC) that is intrinsic to any SDR device, and to avoid signal clipping. Nonetheless, the direct usage of $\hat{E}$ for symbol estimation is not mandatory. For instance, an AGC loop or a double-AGC loop can be designed to rescale the received constellation so that it fits a predefined grid that allows for proper symbol estimation [9]. Thus, the 2r8APSK receiver is slightly more complex than an ordinary 8PSK receiver, and the 2r16APSK receiver has approximately the same complexity of the 16QAM, which subsumes accurate estimation of $E$ or equivalent quantity.

Taking into account only the nearest-neighbor symbols in (4), it can be found an approximate closed form expression for the bit error probability achieved by the 2rMAPSK modulations, for $M=8$ or $M=16$, which is

$$
P_{\mathrm{b}} \approx \frac{1}{\log _{2} M}\left\{\begin{array}{l}
\operatorname{erfc}\left[\sqrt{\frac{E_{b} \log _{2} M}{2 N_{0}}\left(1-\frac{2 \sqrt{z}}{1+z} \cos \frac{2 \pi}{M}\right)}\right] \\
+\operatorname{erfc}\left[\sqrt{\frac{E_{b} \log _{2} M}{(1+z) N_{0}}\left(1-\cos \frac{4 \pi}{M}\right)}\right]
\end{array}\right\},
$$

where $z$ is the optimum one given in Fig. 2, according to the modulation to be analyzed.

\section{PAPR ANALYSIS}

The PAPR is defined here as PAPR $=\max |\tilde{s}(t)| / \mathbb{E}\left\{|\tilde{s}(t)|^{2}\right\}$, where $\tilde{s}(t)$ is the complex envelope of the modulated real signal $s(t)$ and $\mathbb{E}\{\cdot\}$ is the expected value of the argument. The PAPR analysis was carried out with the help of a simulation implemented in VisSim/Comm [10], which is a software tool for modeling and simulating dynamic systems [11]. The real and imaginary parts of the complex envelope of the modulated signals went through low-pass root raised cosine (RRC) pulse shaping filters whose roll-off factors were varied from 0.1 to 1 in steps of 0.05. The filtered signals fed a PAPR measurement block, which outputs the PAPR in decibels. The implemented 
simulation was verified by plotting the PAPR of an RRCfiltered binary phase-shift keying (BPSK) signal, whose result matched [12, Fig. 4].

From the plots in Fig. 6, it can be seen that the proposed 2r8APSK modulation has a PAPR around $2 \mathrm{~dB}$ above the one achieved with the 8PSK, and that the 2r16APSK modulation has a PAPR around $1 \mathrm{~dB}$ below the 16QAM, for roll-off factors above 0.5 . The differences become smaller at lower roll-offs.

As will be demonstrated in the next section, the higher PAPR of the 2r8APSK with respect to the 8PSK establishes a trade-off with an improved performance in terms of BER. Additionally, the lower PAPR of the 2r16APSK with respect to the 16QAM comes with a minor performance penalty.

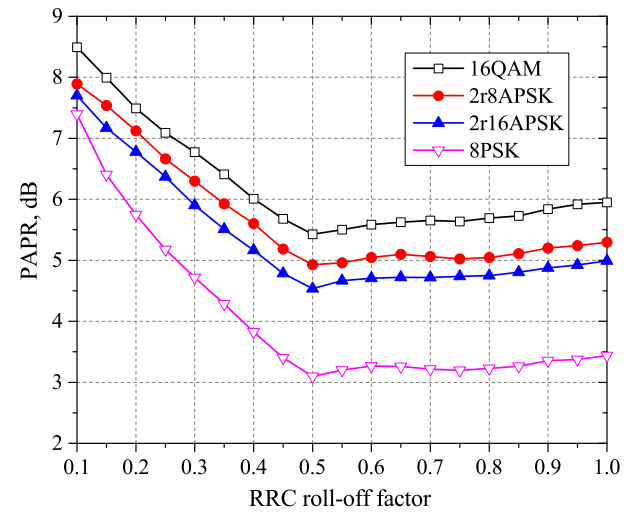

Fig. 6. Peak-to-average power ratio versus RRC filter roll-off factor.

It is interesting to notice in Fig. 6 that the minimum PAPR occurs around a roll-off of 0.5 , and that the PAPR increases slightly above this value. Below it, the PAPR increases dramatically. These phenomena are also in agreement with [12].

\section{BER ANALYSIS}

A Mathcad worksheet was built [10] to support the construction of the 2r8APSK and the 2r16APSK constellations, as well as to assess the BER of the 2r8APSK, the 2r16APSK, the 8PSK and the 16QAM over the AWGN channel. Fig. 7 gives the BER of these modulations. It can be seen that the 2r8APSK outperforms the 8PSK, the difference becoming larger as $E_{\mathrm{b}} / N_{0}$ increases. The theoretical bit error probability of the Gray-mapped 8PSK comes from [8, p. 234], which is

$$
P_{\mathrm{b}} \approx \frac{\sum_{i=1}^{\max (M / 4,2)} \operatorname{erfc}\left\{\sqrt{\frac{E_{\mathrm{b}} \log _{2} M}{N_{0}}} \sin \left[\frac{(2 i-1) \pi}{M}\right]\right\}}{\max \left(\log _{2} M, 2\right)} .
$$

From Fig. 7 it can be observed that the power efficiency provided by the 2r16APSK is not too far from the one achieved with the 16QAM, only $1 \mathrm{~dB}$ below at moderate to high values of $E_{\mathrm{b}} / N_{0} \mathrm{~dB}$. The theoretical performance of the Gray-mapped square 16QAM comes from [8, p. 227], which is given by

$$
P_{\mathrm{b}} \approx \frac{2(\sqrt{M}-1) \sum_{i=0}^{\sqrt{M} / 2-1} \operatorname{erfc}\left[(2 i+1) \sqrt{\frac{3 E_{\mathrm{b}} \log _{2} M}{2(M-1) N_{0}}}\right]}{\sqrt{M} \log _{2} M} .
$$

Fig. 7 also gives the theoretical results obtained via (6) for the 2r8APSK and the 2r16APSK modulations. It can be

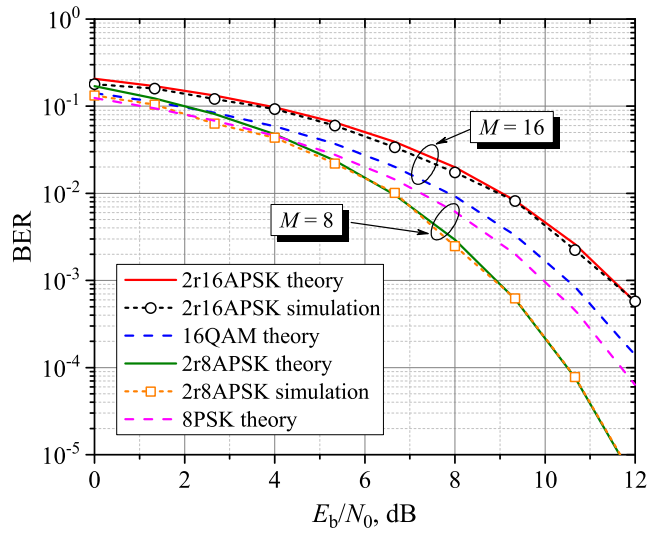

Fig. 7. Bit error rates for the 2r8APSK, 2r16APSK, 8PSK and 16QAM modulations over the AWGN channel.

seen that these results closely approximate the corresponding estimated BERs, mainly at higher values of $E_{\mathrm{b}} / N_{0}$.

\section{CFM ANALYSIS}

The modulations considered in Table I can be easily compared in light of the constellation figure of merit (CFM) [13], which is an indirect measure of their power efficiencies. The CFM is the quotient between the squared minimum Euclidean distance and the average constellation energy. Table II gives the CFM and the radius ratio (when applicable) of the modulations. The radius ratios of the 16APSK are the minimum and maximum values given in [6, Table 9]. Since a higher CFM corresponds to a higher power efficiency, it can be readily established the ranking among the analyzed modulations. Notice that the best 16APSK modulation from [6] could be added to the options of choice, since its CFM does not differ too much from the CFM attained by the 2r16APSK.

TABLE II

CFM AND RADIUS RATIO OF SOME MODULATIONS

\begin{tabular}{|c|c|c|}
\hline Modulation & CFM & Radius ratio \\
\hline 2r8APSK & 0.805 & 1.82 \\
\hline 8PSK & 0.586 & Not applicable \\
\hline Square 16QAM & $0.400[4]$ & Not applicable \\
\hline 16APSK [6], [7] & 0.340 & 2.57 \\
\hline 2r16APSK & 0.325 & 1.61 \\
\hline A16QAM [3], [4] & $0.268[4]$ & Not applicable \\
\hline 16APSK [6], [7] & 0.260 & 3.15 \\
\hline
\end{tabular}

\section{CONCLUSIONS}

The modulations 2r8APSK and 2r16APSK were proposed as alternatives to the 8PSK and the square 16QAM, respectively. The 2r8APSK achieves lower bit error rate than the 8PSK, at the cost of a $2 \mathrm{~dB}$ higher peak-to-average power ratio, and a slightly more complex receiver due to the need of average received symbol energy estimation. The 2r16APSK yields a $1 \mathrm{~dB}$ lower peak-to-average power ratio relative to the 16QAM, with roughly the same receiver complexity, but at the cost of a slightly lower power efficiency, around $1 \mathrm{~dB}$. The modulations 2r8APSK and 2r16APSK, added to the wellknown 8PSK and square 16QAM, increase the possibilities of choice between an 8-ary and a 16-ary signaling scheme, taking into account the bit error rate, the peak-to-average power ratio, and the constellation figure of merit. 


\section{REFERENCES}

[1] D. A. Guimarães, Digital Transmission: A Simulation-Aided Introduction with VisSim/Comm, ser. Signals and Communication Technology. Springer, 2009, doi: 10.1007/978-3-642-01359-1.

[2] C. Thomas, M. Weidner, and S. Durrani, "Digital amplitudephase keying with M-ary alphabets," IEEE Transactions on Communications, vol. 22, no. 2, pp. 168-180, February 1974, doi: 10.1109/TCOM.1974.1092165.

[3] X. Liu and H.-C. Wu, "Analysis and evaluation of novel asterisk-16QAM constellation family and its application for PMEPR control in Golaycoded OFDM systems," in 2010 IEEE International Conference on Communications, May 2010, pp. 1-5, doi: 10.1109/ICC.2010.5502339.

[4] X. Liu and H. Wu, "Novel asterisk 16QAM constellation for COFDM," IEEE Communications Letters, vol. 14, no. 7, pp. 596-598, July 2010, doi: 10.1109/LCOMM.2010.07.100372.

[5] K. P. Liolis and N. S. Alagha, "On 64-APSK constellation design optimization," in 2008 10th International Workshop on Signal Processing for Space Communications, Oct 2008, pp. 1-7, doi: 10.1109/SPSC.2008.4686709.

[6] European Telecommunications Standards Institute (ETSI), "Digital video broadcasting (DVB); second generation framing structure, channel coding and modulation systems for broadcasting, interactive services, news gathering and other broadband satellite applications (DVB-S2)," March 2013. [Online]. Available: http://www.etsi.org/deliver/etsi_en/ 302300_302399/302307/01.03.01_60/en_302307v010301p.pdf.

[7] W. Sung, S. Kang, P. Kim, D.-I. Chang, and D.-J. Shin, "Performance analysis of APSK modulation for DVB-S2 transmission over nonlinear channels," Int. J. Satellite Communications Networking, vol. 27, pp. 295311, 2009, doi: 10.1002/sat.938.

[8] M. Simon and M. Alouini, Digital Communication over Fading Channels, ser. Wiley Series in Telecommunications and Signal Processing. Wiley, 2005, doi: 10.1002/0471715220.

[9] J. Pérez, S. Pueyo, and B. López, Automatic Gain Control: Techniques and Architectures for RF Receivers, ser. Analog Circuits and Signal Processing. Springer, 2011, doi: 10.1007/978-1-4614-0167-4.

[10] D. A. Guimarães, "VisSim/Comm and Mathcad diagrams for assessing the PAPR, the BER and the CFM of the modulations 8PSK, 16PSK, 16QAM, 2r8APSK, 2r16APSK, A16QAM and the DVB's 16APSK," Sep. 2018. [Online]. Available: \{https://www.dropbox.com/ s/m3p6zki424ieoq7/2rMAPSK.zip?dl=0.\}

[11] Altair Engineering, Inc. (former Visual Solutions, Inc.), "VisSim: A graphical language for simulation and model-based embedded development," Nov. 2015. [Online]. Available: http://www.vissim.com/ products/vissim/comm.html

[12] S. Daumont, B. Rihawi, and Y. Lout, "Root-raised cosine filter influences on PAPR distribution of single carrier signals," in 2008 3rd International Symposium on Communications, Control and Signal Processing, March 2008, pp. 841-845, doi: 10.1109/ISCCSP.2008.4537340.

[13] G. D. Forney and L.-F. Wei, "Multidimensional constellations. I. introduction, figures of merit, and generalized cross constellations," IEEE Journal on Selected Areas in Communications, vol. 7, no. 6, pp. 877892, Aug 1989, doi: 10.1109/49.29611.

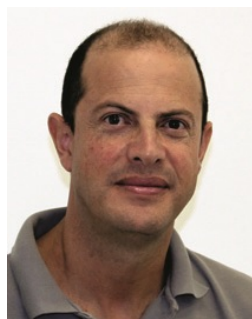

Dayan Adionel Guimarães received the Ph.D. degree in Electrical Engineering from the State University of Campinas, Unicamp, Brazil, in 2003. $\mathrm{He}$ is currently a Lecturer and a Researcher with the National Institute of Telecommunications, Inatel, Brazil. His research interests are fixed and mobile wireless communications, specifically radio propagation, digital transmission, spectrum sensing for cognitive radio, and convex optimization and signal processing applied to communications. 